\title{
Reply to Letter Regarding “Amplatzer Vascular Plugs Versus Coils for Embolization of Pulmonary Arteriovenous Malformations in Patients with Hereditary Hemorrhagic Telangiectasia"
}

\author{
Noam Tau ${ }^{1}$ (1) Eliyahu Atar ${ }^{1} \cdot$ Meir Mei-Zahav ${ }^{2} \cdot$ Gil N. Bachar ${ }^{1} \cdot$ \\ Tamir Dagan ${ }^{3} \cdot$ Einat Birk $^{3} \cdot$ Elchanan Bruckheimer ${ }^{3}$
}

Received: 27 July 2016/Accepted: 1 August 2016/Published online: 9 August 2016

(C) Springer Science+Business Media New York and the Cardiovascular and Interventional Radiological Society of Europe (CIRSE) 2016

To the Editor,

In our article on the recanalization rate of Amplatzer plugs versus coils for transcatheter closure of pulmonary arteriovenous malformations (PAVMs) in HHT, we reported on the retrospective evaluation of available patient data and imaging. As with all retrospective studies, it has the inherent limitations of nonrandomization and patient selection bias.

However, the patients who underwent a repeat pulmonary angiogram procedure did so for clinical reasons such as cyanosis or an increased amount of shunt [bubbles] on contrast echocardiogram or the presence of new PAVMs on a CT, and not just to evaluate the presence of recanalization. Many of these patients had multiple PAVMs embolized with coils or plugs in the same lung and therefore are acting as their own controls in a way.

Noam Tau

taunoam@gmail.com

1 Departments of Diagnostic Imaging, Rabin Medical Center, Beilinson and HaSharon Hospitals, 39 Jabotinski St., 49100 Petach Tikva, Israel

2 Department of Pulmonology, Sackler Faculty of Medicine, Tel Aviv University, Tel Aviv, Israel

3 Sackler Faculty of Medicine, Institute of Pediatric Cardiology, Tel Aviv University, Tel Aviv, Israel
Our study suggests that Amplatzer plugs have a significantly lower recanalization rate than coils. As we have mentioned in our paper, the true recanalization rate of both coils and plugs can only be accurately assessed in a prospective randomized controlled study.

\section{Compliance with Ethical Standards}

Conflict of Interest Elchanan Bruckheimer reports holding the following positions: Medical director, Employee, and ShareholderRealview Imaging, Yokneam, Israel. Consultant-Atrium Medical, New Hampshire USA. Consultant-Valtech, Or Yehuda, Israel. Consultant-Enopace, Cesarea, Israel Medical. Director and Shareholder-Vascular Platforms, Israel. Medical Director and Shareholder-Restore Medical, Israel. All the other authors declare that they have no conflicts of interest.

Informed Consent The study was approved by the Institutional Review Board with a waiver of informed consent. 